\title{
Los retos de la reparación a las víctimas del conflicto armado en Colombia para la construcción de paz ${ }^{1}$ \\ The callenges of reparation to the victims of the armed conflict in Colombia for the construction of peace
}

Rafaela Sayas-Contreras ${ }^{2}$

\section{Resumen}

Los procesos de negociación política del conflicto armado tienen como eje central la reparación de las víctimas, esta tiene varios componentes que le apuestan a la búsqueda de la verdad, justicia, reparación y garantías de no repetición. Sin embargo, estos no pueden ser concebidos de manera retórica, sino planteados desde programas gubernamentales de reparaciones que atiendan una línea de base de sujetos a reparar, mecanismos idóneos para lograrlo, un presupuesto programáticamente asignado y un tiempo para cumplir dichos objetivos. De tal manera que este trabajo busca reflexionar sobre cómo se plantean los procesos de reparación para las víctimas y cuáles son las deudas pendientes en un marco de transición hacia la paz.

Palabras clave: reparaciones, víctimas, desarrollo humano, construcción de paz, posconflicto.

\section{Abstract}

The political negotiation processes of the armed conflict have as central axis the reparation of the victims, this one has several components that bet to the search of the truth, justice, reparation and guarantees of non-repetition, however, these can not be conceived In a rhetorical way, but it raised from government reparations programs that meet a baseline of subject to be repair, appropriate mechanisms to achieve it, programmatically assigned a budget and a time to meet those objectives. Thus, this work seeks to reflect on how the reparation processes are presented for the victims and what are the outstanding debts with them in a framework of transition towards peace

Keywords: reparations, victims, human development, peacebuilding, post-conflict.

1 Proyecto "Observatorio de Reparaciones". Adelantado por el Grupo Conflicto y Sociedad de la Universidad de Cartagena, presentado para el XI Encuentro de la Red de Grupos y Centros de Investigación Jurídica y Sociojurídica Nodo Caribe.

2 Docente Investigadora de la Universidad de Cartagena, Investigadora Asociada Colciencias, Directora del Observatorio Regional de Reparaciones Colectivas para Víctimas (Universidad de Cartagena-Colombia, CODHES-USAID). Directora del Grupo de Investigaciones Conflicto y Sociedad. rafaelaester@gmail.com 


\section{INTRODUCCIÓN}

Colombia tras un conflicto de más de 50 años, en noviembre de 2016, concluyó un proceso de negociación política con la firma del acuerdo final que inició en La Habana en el año 2012 entre el Gobierno colombiano y el movimiento guerrillero FARC-EP, acuerdo que incluye el desarme, desmovilización reincorporación de los excombatientes y el sometimiento de estos a una Jurisdicción Especial de Paz (JEP) para los que reconozcan las responsabilidades por violaciones del derecho internacional de los derechos humanos e infracciones al derecho internacional humanitario. Sin embargo en este contexto político, cabe preguntarse, ¿hay reparación para las víctimas? ¿cómo se plantean los procesos de reparación integral para víctimas del conflicto armado colombiano? y ¿cuáles son las deudas pendientes con las víctimas por los hechos victimizantes? Aspecto importante en la medida que la transición hacia una sociedad pacificada, es que estén dadas las condiciones para la sostenibilidad de la paz y la resolución pacífica de conflictos, de tal manera que para abordar este trabajo, en primer lugar se hará una aproximación conceptual del tema a manera de marco referencial, luego se analizará la relación entre víctimas y reparación y por último la importancia de estos temas para el posconflicto y se plantearán las conclusiones.

\section{METODOLOGÍA}

Se trata de una investigación cualitativa, descriptiva, de tipo analítico, documental, que se desarrollará a nivel teórico; la cual busca analizar el contexto sociopolítico del país, de cara a la valoración de la reparación y los mecanismos institucionales para su otorgamiento. Las fuentes utilizadas son de carácter secundario, las cuales se relevaron, organizaron y analizaron tomando como categorías de análisis la victimización y la reparación.

\section{APROXIMACIÓN CONCEPTUAL}

El pasado nos ha señalado que el problema de los migrantes forzados y su reflejo en las políticas públicas en Colombia, más bien, ha sido reciente, aun cuando el conflicto armado se instaló de manera permanente en el territorio desde final de los años 80 . El padecimiento de hechos victimizantes por parte de la población civil pasó desapercibido y solo hasta 1997 se expide una normativa relacionada con la protección de los migrantes forzados (L. 386) que sirvió de base para que en el año 2004 la Corte Constitucional colombiana declarara sobre el desplazamiento un estado de cosas inconstitucional. Sobre el particular cabe decir que, para esa época no se planteaba el otorgamiento de reparaciones para las víctimas, de hecho el único hecho victimizante reconocido para los migrantes forzados era el desplazamiento forzado y las pocas condenas al Estado eran precedidas por tortuosos procesos administrativos de reparación directa y a través de fallo judicial.

Quiere decir ello que las normas de protección en este momento, solo aludían a la prevención y acompañamiento para los desplazamientos forzados y el reasentamiento o retorno de estas poblaciones, pero solo al amparo de los gobiernos nacional/local, aspecto que contrasta con la protección de esos mismos migrantes forzados que quedaban cobijados por normativas internacionales sobre refugio, suficientemente regulados por la Convención de Ginebra.

Así las cosas, más allá de que Naciones Unidas profiriera los Principios Deng - Principios para las personas que padecían desplazamientos forzados internos, estas no se consideraban vinculantes, pero hay que dar cuenta que la norma interna de protección para los desplazados en Colombia, contiene las mismas prerrogativas que para los migrantes forzados internos tienen los principios Deng.

Hablar de desplazamiento en Colombia, es referirse a la cadena de victimizaciones a los que se ha expuesto la población civil en contextos de violencia generalizada, con la particularidad que en el territorio, la seguridad, o más bien la falta de ella, es un cúmulo de diversos factores de carácter estructural, a los que se suman las vulnerabilidades ocasionadas por la violencia directa.

El fenómeno de las migraciones y de los desplazamientos forzados, hoy es un tema de agenda global, de hecho algunos autores, por ejemplo Vidal (2007) plantean que, justamente la preocupación por el ingreso desordenado y sin reglamentación de personas foráneas, no deseadas a los países, determina ciertas políticas de contención a nivel internacional para que no se produzcan migraciones "no deseadas"; para ello existe un control de las migraciones que se expresa en el derecho como orden y en parámetros de focalización de ayudas de organismos de cooperación internacional a los desplazados internos de los países, con el fin de que permanezcan dentro de las fronteras de sus respectivos Estados. 
Dentro de las migraciones forzadas cabe incluir las migraciones forzadas internas. Los desplazados internos son personas que se encuentran en situación como la de los refugiados, pero que no han cruzado una frontera internacional. Al incluir la categoría de desplazados dentro de los conceptos de migraciones globales, se pretende mostrar que las personas desplazadas hacen parte de los mismos procesos que constituyen migraciones forzadas internacionalmente que pueden llevar al refugio, y que la atención de las mismas es tan relevante para la comunidad internacional como puede serlo la de las migraciones legales e ilegales, por cuanto constituyen una parte significativa de las migraciones en el mundo -actualmente la más grande- $y$ con ello introducen presión sobre las corrientes migratorias internacionales. (Vidal, 2007, p.39).

Retomando el tema de los desplazamientos forzados, tomemos en consideración por una parte, el análisis de la naturaleza de las normativas que regulan cada fenómeno y las jurisdicciones que cobijan las respectivas poblaciones. Para los refugiados se encuentra previsto un estatuto que adopta la forma de tratado internacional, suscrito por ministros plenipotenciarios de los Estados firmantes, posteriormente sometido a ratificación por cada Estado conforme a su derecho interno, de donde surge su fuerza vinculante. En este sentido, es regulado por el Derecho Internacional, por lo tanto impone obligaciones multilaterales a los Estados que libremente lo suscribieron.

En ese orden de ideas, pasa lo contrario con los Principios Rectores de Desplazamiento, al ser normativas supranacionales pero que solo constituyen guías o pautas recopiladas por la Organización de Naciones Unidas para promover la ayuda humanitaria de gobiernos y organizaciones internacionales hacia la población en condición de desplazamiento interno. Con lo cual, es predicable el carácter no vinculante de tal recopilación de principios. En este aspecto, en el caso de Colombia, se hace necesario precisar que la Corte Constitucional colombiana consideró mediante la Sentencia T-025 de 2004, que los principios Deng o Principios Rectores del Desplazamiento Forzado, debían ser considerados en la interpretación de las normas que consagran un derecho fundamental para los desplazados:

Es por ello que en la Sentencia T-025 de 2004, la Corte afirmó que

en la interpretación de las normas que consagran o desarrollan

un derecho fundamental de las personas que han sido despla- zadas, debe tenerse en cuenta: (1) los principios de interpretación y aplicación contenidos en la Ley 387 de 1997 (II) los Principios Rectores de los Desplazamientos Internos; (III) el principio de favorabilidad en la interpretación de las normas que protegen a la población desplazada; (IV) el principio de buena fe y el derecho a la confianza legítima; $y(v)$ la prevalencia del derecho sustancial propio del Estado Social de Derecho.

En lo tocante a los sujetos pasivos (debitum), en razón de las obligaciones que emanan de los respectivos instrumentos, existen también notorias diferencias. En el caso del refugio, los obligados son los Estados como sujetos de derecho internacional. Libremente ratificaron, consecuencialmente adquirieron obligaciones impositivas con respecto de los sujetos que encontrándose en la situación reglada así lo solicitaren. Dicho de otra manera, la solicitud de refugio obliga a un Estado frente a otro en términos de reciprocidad, cuando sus ciudadanos así lo pidieren. Para los desplazados internos la situación es diferente. Al no revestir los Principios Rectores de Desplazamiento la forma de un tratado, no obligan sus preceptos. Luego, la protección legal de los grupos desplazados debe ser proporcionada por el derecho interno de cada Estado he ahí la injusticia en el tratamiento de personas que encuadrándose en el mismo supuesto y padeciendo las mismas circunstancias quedan ubicadas en sistemas de protección distintos.

Nótese que hasta este momento en Colombia solo se habla de desplazamiento forzado. Solo hasta 2005, de cara al proceso de desmovilización de las Autodefensas Unidas de Colombia (AUC) se expide la Ley 975, que ilustra los procesos de dejación de armas de estos grupos y el sometimiento a la justicia de los mismos a través de mecanismos de justicia transicional. Es en este compendio normativo que se define por vez primera quién es una victima, definición que es ampliada con posterioridad en la Ley de Víctimas y Restitución de Tierras, que constituye la apuesta del Gobierno de Juan Manuel Santos desde que se posesionó como presidente en el año 2010.

\section{REPARACIONES Y VICTIMAS}

La Asamblea General de las Naciones Unidas, mediante la Resolución A/RES/60/147 del 24 de octubre de 2005, aprobó los "Principios

\footnotetext{
3 Corte Constitucional Colombiana, Sentencia T-742 de 2009, magistrado ponente Luis Emesto Vargas Silva.
} 
y directrices básicos sobre el derecho de las víctimas de violaciones de las normas internacionales de derechos humanos y del Derecho Internacional Humanitario a interponer recursos y obtener reparaciones" estos principios están contenidos en el documento (E/CN.4/2005/59), relacionados con las normativas que regulan el derecho de las víctimas a obtener reparaciones por violaciones sufridas por el incumplimiento de las obligaciones de los Estados.

Aun cuando el reconocimiento de las victimizaciones desde una perspectiva social e institucional en Colombia son tardías, en el marco de las políticas públicas, se han acogido en el derecho interno, normativas que definen quién es una víctima y se establecen programas gubernamentales de otorgamiento de ayudas en condiciones de emergencia; más recientemente en 2011, los programas gubernamentales de reparaciones, apuestan por la restitutio in integrum, que abarca una reparación integral a partir de los principios de verdad, justicia, reparación y garantías de no repetición.

El pleno restablecimiento de las obligaciones de respeto y garantía requiere un complejo diseño de medidas de reparación que tiendan, no solo a borrar las huellas que el hecho anticonvencional ha generado, sino también comprensivo de las medidas tendientes

a evitar su repetición. (Rousset, 2011, p.65)

Los derechos de las víctimas han ido posicionándose como tema de agenda política en Colombia, de la mano del reconocimiento de los daños padecidos en el marco del Derecho Internacional de los Derechos Humanos y del Derecho Internacional Humanitario. La reparación para que cumpla el propósito de dignificación a las víctimas tiene que ser integral, pero ¿qué significa esta connotación de integralidad? Para responder esto, hay que afirmar que si bien la reparación tiene varios componentes que le apuestan a la búsqueda de la verdad, justicia, reparación y garantías de no repetición, estos no pueden ser concebidos de manera retórica, sino planteados desde programas gubernamentales de reparaciones que atiendan una línea de base de sujetos a reparar, mecanismos idóneos para lograrlo, un presupuesto programáticamente asignado y un tiempo para cumplir dichos objetivos. A continuación vamos a referirnos a cada uno de ellos.
Respecto de la línea de base de víctimas, es un tema que se ha ido consolidando paulatinamente con la creación de la institucionalidad propia y la tecnificación de procesos. La Ley 1448 creó el Sistema Nacional de Atención y Reparación Integral a las Víctimas (SNARIV) que corresponde con un proceso político diferente creando una ruta de reparación integral para las víctimas de conformidad con la ley que busca,

[r]eparar de manera integral, adecuada, diferenciada, transformadora y efectiva a las víctimas, por el daño sufrido como consecuencia de las violaciones a los derechos humanos e infracciones al Derecho Internacional Humanitario en Colombia. Para tal fin contempla las medidas de restitución, indemnización, rehabilitación, satisfacción y garantías de no repetición.

El inicio de la ruta de reparación integral es precedido por la declaración de víctima de los hechos victimizantes ante las oficinas del Ministerio Público (Personerías Municipales, Defensoría del Pueblo o Procuraduría) y luego solicitar su inclusión en el Registro Único de Víctimas, salvo que ya hubiese declarado con anterioridad, antes de entrar en vigencia la ley, en este caso no debe hacer una nueva declaración, pero si quiere puede ampliar los hechos victimizantes si los hubiere sufrido.

Frente a las prerrogativas establecidas en favor de las víctimas, dos de ellas contienen un reto para la política pública en Colombia y que implican una apropiación presupuestal significativa, la restitución de tierras y la indemnización por vía administrativa, como componentes de la reparación del daño causado, no por ello las demás dejan de tener importancia, atendiendo e carácter de integralidad de la misma. Así las cosas, la línea de base total de víctimas, según datos de la Unidad de Atención y Reparación Integral a las Víctimas (UARIV) con corte a 2017, es de 8.376.463 víctimas registradas, de estas 302.191 fueron incluidas por fallo judicial y la diferencia, es decir, 9.074.272 por solicitud directa ante la UARIV.

Por otra parte las cifras exclusivamente de desplazamiento forzado por año, las podemos apreciar en la Tabla 1, la cual referencia el número de personas expulsadas, recibidas y declaradas, y del número total de aquellas que, habiéndose desplazado no acudieron a las autoridades respectivas: 
Tabla 1. Desplazamiento - Personas. Total Nacional

\begin{tabular}{|c|c|c|c|}
\hline Vigencia & Personas Expulsadas & Personas Recibidas & Personas declaradas \\
\hline Antes de 1985 & 75.450 & 6.281 & 79.721 \\
\hline 1985 & 13.322 & 10.478 & 2 \\
\hline 1986 & 14.905 & 12.133 & 2 \\
\hline 1987 & 18.539 & 14.742 & 2 \\
\hline 1988 & 32.757 & 25.306 & 7 \\
\hline 1989 & 29.058 & 22.760 & 25 \\
\hline 1990 & 36.874 & 30.124 & 286 \\
\hline 1991 & 32.402 & 26.920 & 4 \\
\hline 1992 & 42.948 & 35.892 & 24 \\
\hline 1993 & 48.593 & 40.773 & 18 \\
\hline 1994 & 52.987 & 44.293 & 28 \\
\hline 1995 & 103.937 & 89.275 & 361 \\
\hline 1996 & 135.589 & 109.404 & 3.431 \\
\hline 1997 & 244.099 & 211.534 & 16.036 \\
\hline 1998 & 236.283 & 214.319 & 60.512 \\
\hline 1999 & 268.122 & 225.719 & 49.967 \\
\hline 2000 & 585.914 & 570.190 & 328.810 \\
\hline 2001 & 648.407 & 635.877 & 437.927 \\
\hline 2002 & 746.905 & 723.805 & 520.686 \\
\hline 2003 & 448.128 & 436.031 & 272.360 \\
\hline 2004 & 411.029 & 407.055 & 254.421 \\
\hline 2005 & 469.660 & 468.041 & 312.407 \\
\hline 2006 & 450.801 & 459.326 & 375.830 \\
\hline 2007 & 472.976 & 488.591 & 458.757 \\
\hline 2008 & 421.938 & 447.967 & 502.918 \\
\hline 2009 & 245.595 & 271.492 & 418.876 \\
\hline 2010 & 189.481 & 207.830 & 358.988 \\
\hline 2011 & 227.801 & 244.233 & 436.091 \\
\hline 2012 & 228.689 & 241.430 & 519.756 \\
\hline 2013 & 250.485 & 262.962 & 717.822 \\
\hline 2014 & 239.974 & 252.924 & 760.683 \\
\hline 2015 & 164.927 & 172.525 & 641.945 \\
\hline 2016 & 63.238 & 65.909 & 111.970 \\
\hline 2017 & 106 & 114 & 392 \\
\hline Sin información & 4.308 & 205.263 & 63 \\
\hline
\end{tabular}

Fuente: Unidad de Atención y Reparación Integral a Víctimas (UARIV). Fecha corte marzo 1, 2017
Los datos numéricos aluden a personas víctimas oficialmente registradas y solicitantes bien sea de atención o inclusión en ruta de reparación, para esta última, según el hecho victimizante el valor entregado como compensación económica puede ascender a 40 salarios mínimos legales mensuales, que equivalen actualmente a $\$ 29.508 .680$ mil pesos colombianos, 010.538 dólares aproximadamente. De hecho, según la ley los hechos victimizantes son: homicidio, desaparición forzada, secuestro, lesiones personales que generan incapacidad permanente o que solo generan incapacidad temporal, reclutamiento de niños, niñas y adolescentes (NNA), delitos contra la libertad e integridad sexual, incluidos niños, niñas y adolescentes nacidos como consecuencia de una violación sexual en el marco del conflicto armado, tortura y demás tratos crueles e inhumanos y desplazamiento forzado, es decir, nueve hechos victimizantes:

Tabla 2. Hechos victimizantes

\begin{tabular}{|c|c|c|}
\hline HECHO VICTIMIZANTE & VALOR INDEMNIZACIÓN & CARACTERISTICA \\
\hline Homicidio & 40 SMLMV & Se distribuye a total de familiares y/o cónyuge \\
\hline Desaparición forzada & 40 SMLMV & Se distribuye a total de familiares y/o cónyuge \\
\hline Secuestro & 40 SMLMV & Directamente al secuestrado \\
\hline Lesiones permanentes & 40 SMLMV & A quien las sufrió \\
\hline Lesiones temporales & 30 SMLMV & A quien las sufrió \\
\hline $\begin{array}{l}\text { Reclutamiento llíito de menores } \\
\text { (NNA) }\end{array}$ & 30 SMLMV & A quien lo sufrió \\
\hline Delitos sexuales & 30 SMLMV & A quien lo sufrió \\
\hline Torturas y demás tratos crueles & 30 SMLMV & A quien las sufrió \\
\hline Desplazamiento forzado & 27 SMLMV y otros que recibirán 17 SMLMV & Distribuido en núcleo familiar \\
\hline
\end{tabular}

Fuente: Elaboración propia con fundamento en la norma

Como se puede apreciar, el pago de indemnizaciones se asegura con un presupuesto bastante significativo, que hasta el momento es un compromiso que ha venido cumpliendo el Gobierno colombiano, en el marco específico de la Ley de Victimas que es de 10 años, es decir, hasta 2021

\section{REPARACIONES, CONSTRUCCIÓN DE PAZ Y POSCONFLICTO}

Como se planteó con anterioridad, la Ley 1448 aunque define el concepto de reparación integral y señala las rutas de reparación (individuales y colec tivas) no lo es menos el reto de armonizar toda la legislación vigente con los parámetros establecidos en el acuerdo final, específicamente el relacionado con el punto de víctimas y desarrollo rural integral; sobre este último aspecto, 
el acuerdo implica la expresión de decisiones políticas, pero estas deben volcarse en normativas especiales vía fast track.

Las reparaciones para las víctimas, en una transición hacia la pacificación, deben tener la potencialidad de producir transformaciones sociales, es decir, procurar el desarrollo humano en las comunidades, sobre todo las que se relacionan con el otorgamiento de DESC. Uno de los propósitos de la política pública de paz, es asegurar y garantizar el acceso a bienes y servicios, que contribuirían al establecimiento de una paz estable, duradera y sostenible, de cara a las verdaderas transformaciones sociales e inclusión de la población más pobre, no en vano, en el texto final de los acuerdos ocupa un lugar preponderante el punto referido al desarrollo agrario integral que no solo implica la restitución de tierras y la conformación de un banco de tierras, sino el otorgamiento de derechos sociales a la población campesina.

Hoy el reto de las políticas públicas para el desarrollo individual y social de capacidades es alto, teniendo en cuenta que si bien como Estado Social de Derecho se ha apostado por una cobertura universal en algunos derechos como la salud y la educación, estos logran cumplirse en ciudades principales pero no en ciudades secundarias y mucho menos, en territorio rural; en ese sentido aún no se han removido los obstáculos para disfrutar el acceso a derechos de la población excluida en Colombia, aspecto que se agrava cuando se trata de población excluida, pero que también sufrió victimizaciones por la guerra.

Cambiar las normas para construir tolerancia y profundizar la cohesión social es también un aspecto necesario, y a menudo pasado por alto, cuando se habla de la construcción de sociedades resilientes. Las sociedades más cohesivas son más aptas para proteger a las personas contra la adversidad y están en mejor disposición para aceptar las políticas basadas en el principio de universalidad. La falta de cohesión social se correlaciona con el conflicto y la violencia, especialmente en las situaciones de desigualdad en el acceso a los recursos o a los beneficios de la riqueza natural, y con la incapacidad para abordar eficazmente el cambio social o económico rápido o el impacto de las crisis económicas o relacionadas con el clima. En efecto, la consecución de los objetivos generales de la equidad, la inclusión y la justicia refuerza las instituciones sociales y, a su vez, profundizan la cohesión social (Naciones Unidas, Informe sobre Desarrollo Humano, 2014).
Como se ha venido planteando, los retos políticos para el Gobierno colombiano de cara a las transiciones hacia la paz es bastante alto, no solo en lo presupuestal, existen otros aspectos que deben ser considerados como por ejemplo, el marco temporal de la Ley de Víctimas para la armonización de esta normativa con los textos del acuerdo de paz, las mesas de negociaciones con otros movimientos armados como por ejemplo el ELN, la eficiencia de los procesos de desarme y desmovilización, la sostenibilidad de la reincorporación de excombatientes para que no alimenten estructuras criminales ordinarias, la implementación de la Jurisdicción Especial de Paz y la participación política de víctimas y partidos políticos regularizados para los grupos que se reincorporen, entre otros.

\section{CONCLUSIONES}

Retomando los cuestionamientos preliminares planteados en esta ponencia sobre ¿hay reparación para las víctimas? Ha quedado establecido que si bien ha sido tardío y dificultoso el tema, hoy hablar de reparaciones es común, hay una apropiación de la base social sobre el mismo, normativas suficientes para su garantía y otorgamiento, la coyuntura difícil siempre es la implementación. La UARIV paulatinamente ha cumplido con entrega de reparaciones por vía administrativa, aun cuando haya territorios que estén pendientes, el tema en este caso es presupuestal. Normativamente Colombia sigue los parámetros internacionales orientados a que las reparaciones sean transformadoras según los lineamientos de Naciones Unidas.

Respecto de ¿cómo se plantean los procesos de reparación integral para victimas del conflicto armado colombiano? Existe una institucionalidad suficiente como la UARIV -ya mencionada- con procedimientos establecidos para transitar rutas en el marco de programas de reparación administrativo (individual y colectivo), sin que sea óbice para el pago de la reparación, en los casos que se demandó la misma ante las autoridades judiciales (sentencias), y por último, refiriéndonos a las deudas pendientes con las víctimas por los hechos victimizantes, vemos que si bien, se ha trabajado mucho el tema, faltaría una transformación social, que desborda el marco normativo de la Ley de Victimas, pero que por lo menos políticamente está planteada en el acuerdo final con la transformación integral del campo colombiano. Desafío un tanto dificil. 


\section{REFERENCIAS BIBLIOGRÁFICAS}

Congreso de la República De Colombia. Ley 1448 de 2011. Ley de Victimas y Restitución de Tierras.

Corte Constitucional de Colombia. Sentencia T-025 de 2004

Naciones Unidas. Informe sobre Desarrollo Humano 2014. http://www.undp.org/ content/dam/undp/library/corporate/HDR/2014HDR/HDR-2014-Spanish.pdf Rousset Siri, A. (2011). "El concepto de reparación integral en la jurisprudencia de la Corte Interamericana de Derechos Humanos". Revista Internacional de Derechos Humanos, (1), 59-79. http://www.corteidh.or.cr/tablas/usuario/frank/30948.pdf

Van Boven, T. (2010). Principios y directrices basicos de las Naciones Unidas sobre el derecho de las victimas de violaciones manifiestas de las normas internacionales de derechos humanos y de violaciones graves del Derecho Internacional Humanitario a interponer recursos y obtener reparaciones.

Vidal, R. (2007). Derecho global y desplazamiento interno. Creación, uso y desapa. Bogotá: Editorial Pontificia Universidad Javeriana.
Situación jurídica de la mujer colombiana en el post-acuerdo desde la perspectiva del enfoque de género

Legal situation of colombian women in the post-agreement from the perspective of the gender approach Saby Lorena Bravo-Alvarez' - Katrina González-Avilez ${ }^{2}$

Resumen

En el actual proceso de implementación del acuerdo final firmado el pasado noviembre de 2016 entre la

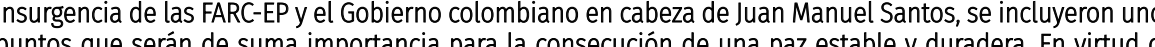
lo anterior este documento tiene como fin analizar y estudiar el enfoque de género, el cual es trasvers a todo lo pactado, y en ello vistumbrar de forma clara el papel e importancia en la práctica que se le esta la ha sumido el conflicto social del pais.

Palabras clave: mujer, Post-acuerdo, enfoque de género, conflicto interno, acuerdos de paz

Abstract

In the current process of implementation of the final agreement signed last november (2016) between FARC's EP insurgency and Colombian government in head of Juan Manuel Santos, included points that Wir be great important for the construction of a stable and lasting peace. Under the above, this document is
intended to analyze and study the gender a paproach which is transversal to everything agreed; and also to see clearly the role and real importance in the practice that is giving to woman. In order to close the gap

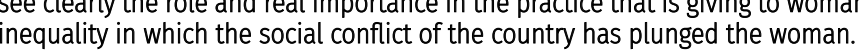

Keywords: women, Post-greement gender focus, internal conflict peace accords.

Estudiante de Vil semesstre de Derecho Vespertino de la Facultad de Derechoy C Ciencias Politicas, de la Universidad de Cartagena, sabylorena_12@hotmail.com

2 Estudiante de VIII semestre de Derecho Vespertino de la Facultad de Derecho y Ciencias Politicas, de la Universidd de Catogen Colombia. Miembro del Semillero de Investigación Reorganización Empresarail dirigido por el professor Andrés Alarcón Lorä ktrinagon@gmail.com 